\title{
Perfil epidemiológico da tuberculose em um município prioritário de Pernambuco no
} período de 2015-2020

\author{
Epidemiological profile of tuberculosis in a priority municipality of Pernambuco in the period
} 2015-2020

\section{Perfil epidemiológico de la tuberculosis en un municipio prioritario de Pernambuco en el período}

2015-2020

\begin{abstract}
Resumo
Objetivo: Avaliar o perfil epidemiológico da tuberculose no município de Recife no período de 2015 a 2020. Metodologia: Trata-se de um estudo retrospectivo, descritivo com abordagem quantitativa sobre o perfil epidemiológico dos casos de tuberculose no município do Recife no período de 2015 a 2020, com dados coletados no Sistema de Notificação de Agravos de Notificação no período de dezembro de 2021 a janeiro de 2022. Resultados: Foram notificados 15.181 casos de tuberculose no município de Recife no período estudado, com predominância do sexo masculino $(68,1 \%)$, faixa etária dos 20-39 (42,85\%), com a forma clínica pulmonar em evidência (79,4\%), sendo que a maioria do modo de entrada de enquadrando como casos novos $(71,9 \%)$ e com a saída do quadro de tuberculose como cura $(47,6 \%)$. A relação da tuberculose com outras patologias e condições sociais dos acometidos ficou bastante evidente. Conclusão: Ficou evidente que a tuberculose ainda é bastante presente no município, ações de educação em saúde e combate a abandono são importantes para diminuição da sua prevalência.
\end{abstract}

Palavras-chave: Tuberculose; Epidemiologia; Atenção à saúde. 


\begin{abstract}
Objective: To evaluate the epidemiological profile of tuberculosis in the city of Recife from 2015 to 2020. Methodology: This is a retrospective, descriptive study with a quantitative approach on the epidemiological profile of tuberculosis cases in the city of Recife from 2015 to 2020, with data collected in the Notifiable Diseases Notification System from December 2021 to January 2022. Results: There were 15,181 cases of tuberculosis reported in the city of Recife during the study period, with a predominance of males $(68.1 \%)$, age group 20-39 (42.85\%), with the clinical pulmonary form in evidence $(79.4 \%)$, with the majority of the entry mode being new cases $(71.9 \%)$ and with leaving tuberculosis as a cure $(47.6 \%)$. The relationship of tuberculosis with other pathologies and social conditions of those affected was quite evident. Conclusion: It was evident that tuberculosis is still quite present in the city, health education actions and combating abandonment are important to reduce its prevalence.
\end{abstract}

Keywords: Tuberculosis; Epidemiology; Delivery of Health Care.

\title{
Resumen
}

Objetivo: Evaluar el perfil epidemiológico de la tuberculosis en la ciudad de Recife de 2015 a 2020. Metodología: Se trata de un estudio descriptivo retrospectivo con un enfoque cuantitativo sobre el perfil epidemiológico de los casos de tuberculosis en la ciudad de Recife de 2015 a 2020, con datos recolectados en el Sistema de Notificación de Enfermedades Notificables de diciembre de 2021 a enero de 2022. Resultados: Se notificaron 15.181 casos de tuberculosis en la ciudad de Recife durante el período de estudio, con predominio del sexo masculino (68,1\%), grupo etario 20-39 (42,85\%), con la forma clínica pulmonar en evidencia $(79,4 \%)$, siendo la mayor parte de la vía de entrada casos nuevos $(71,9 \%)$ y dejando la tuberculosis como cura $(47,6 \%)$. La relación de la tuberculosis con otras patologías y condiciones sociales de los afectados fue bastante evidente. Conclusión: Se evidenció que la tuberculosis aún está bastante presente en la ciudad, las acciones de educación para la salud y el combate al abandono son importantes para reducir su prevalencia.

Palabras clave: Tuberculosis; Epidemiología; Atención a la Salud.

\section{Introdução}

A tuberculose (TB) é uma doença infecto-contagiosa causada pela bactéria Mycobacterium tuberculosis, também conhecida como bacilo de Koch, a doença atinge principalmente os pulmões, contudo em casos mais grave pode afetar outros locais do corpo, como os ossos, laringe, meninges, rins, intestinos, entre outros (Brasil, 2020). Uma pessoa infectada com o bacilo pode apresentar a TB a qualquer momento da vida, sendo os dois primeiros anos o período de maior risco (Espírito Santo, 2015).

A transmissão ocorre principalmente quando uma pessoa com a forma mais contagiosa da TB, a pulmonar, e não esteja em tratamento, expele gotículas contendo o bacilo, quando tosse, fala ou espirra (Bertolozzi et. al. 2014). O diagnóstico é realizado pela avaliação clínica do paciente, e também por meio de exames laboratoriais, como a baciloscopia, o teste rápido e o teste de sensibilidade, outros exames laboratoriais são usados para a avaliação de forma extrapulmonares da TB, como a adenosina deaminase, que é eficiente para o diagnóstico da forma meníngea, a radiografia do tórax também é uma ferramenta eficiente para o seu diagnóstico (Guia de vigilância de saúde, 2019).

A registro da TB no período pré-histórico, onde foram encontrados registros da doença em ossos de múmias com mais de três mil anos, na Europa durante a urbanização crescente houve a disseminação da doença pelo continente, durante esse período a TB ganhou o nome de "peste branca". No Brasil, os primeiros registros da doença datam no período da colonização portuguesa, acreditando-se que o padre jesuíta Manoel de Nóbrega foi o primeiro portador da doença no Brasil, o contato dos índios com os infectados, principalmente nos momentos de "catequização" pelos padres jesuítas, proporcionou o adoecimento e a morte de muitos deles pela TB (Maciel et. al., 2012). Assim como na Europa, a doença se disseminou pelo Brasil, principalmente nos grandes centros urbanos, estima-se que na metade do século 18 a mortalidade era 1/150 habitantes (Campos \& Pianta, 2001).

O combate à TB no Brasil se iniciou pelo trabalho da Liga Brasileira Contra a Tuberculose (atual Fundação Ataulpho de Paiva) e da Liga Paulista Contra a Tuberculose. As ligas prestava ações para profilaxia e tratamento de acordo com os métodos científicos da época, que pautava principalmente em campanhas de educação sanitária, implantação de sanatórios, dispensários e preventórios, as ligas também foram responsáveis pelo início da vacinação com a BCG, no ano de 1927 (Hijjar et. al., 2007$).$ 
A partir da década de 1940 surgiu o Serviço Nacional de Tuberculose, que entre as suas principais ações criou a Campanha Nacional Contra Tuberculose (CNCT), que dentre as suas ações destaca-se a padronização do tratamento contra a doença, tornar a vacina BCG obrigatória, incluí-la na lista de doenças com notificação compulsória, além de tornar gratuito os meios de prevenção, diagnóstico e tratamento da doença.

Atualmente a TB é um problema de saúde pública no Brasil, sendo considerado um dos países prioritário para o controle da doença segundo a Organização Mundial da Saúde (OMS), devido ao fato da sua frequente inclusão na lista dos 30 países com alta carga para infecção com a TB e para co-infecção TB/AIDS (WHO, 2020). Devido a isso, o Governo Federal elaborou o Programa Nacional de Controle da Tuberculose (PNCT), com a função de reduzir a prevalência da TB na população brasileira.

No ano de 2019 houve 96.655 casos notificados de TB no Brasil, desses 25.058 foram na região nordeste, sendo o estado de Pernambuco apresentando o maior número de casos da região (6.151), em âmbito nacional o estado ficou em quarto lugar em casos de TB notificados, ficando atrás apenas de São Paulo (22.025), Rio de Janeiro (14.967) e Rio Grande do do Sul (7.530). Por sua importância epidemiológica, o Governo Estadual de Pernambuco incluiu a TB na lista das sete doenças negligenciadas do Plano Integrado de Ações para o Enfrentamento às Doenças Negligenciadas, o Programa Sanar (Pernambuco, 2019)

Em 2019 cidade do Recife (2.567) foi responsável por uma boa parcela nos números de casos da TB no estado de Pernambuco, por esse motivo foi incluído na lista das cidades prioritários da doença do estado Programa Sanar, no quadriênio de 2019-2022

Perante o exposto, é de suma importância a realização de estudos epidemiológicos sobre a tuberculose em cidades com alta prevalência da doença, para compreender os fatores que contribuem para a sua continuidade, e também para que gestores e profissionais de saúde busquem ações para o controle, prevenção e tratamento da doença. O objetivo deste trabalho foi avaliar o perfil epidemiológico da tuberculose na cidade do Recife no período de 2015 a 2020

\section{Metodologia}

Trata-se de um estudo retrospectivo, descritivo, com abordagem quantitativa (Hochman et. al. 2005). Foram utilizados dados secundários do Sistema de Informações de Agravos de Notificação (SINAN), mediante ao acesso do Tabulador Genérico de Domínio Público (TABNET), na seção “epidemiologia e morbidade", partindo para o tópico "tuberculose". A coleta de dados foi realizada no mês de dezembro de 2021.

A cidade do Recife é localizada na região nordeste do Brasil, capital do estado de Pernambuco. O município possui uma área total de $218.843 \mathrm{~km}^{2}$ e uma população estimada para o ano de 2021 de 1.661 .017 habitantes, possuindo uma densidade média de 7.039.64 hab $/ \mathrm{km}^{2}$ (Instituto Brasileiro de Geografia e Estatística, 2021).

Para alcançar o objetivo proposto do trabalho foram analisados as variáveis, número de casos no período estudado, sexo, faixa etária, modo de entrada e de saída, forma clínica, associação com a AIDS, alcoolismo, tabagismo e diabtes e os casos entre a população privada da liberdade e as que vivem em situação de rua. As bases de dados Google Acadêmico e a Scientific Electronic Library Online (SCIELO) foram utilizadas para a realização da pesquisa bibliográfica. A análise estatística descritiva foi realizada com o auxílio do programa Microsoft Excel 2010.

\section{Resultados e Discussão}

A análise dos dados epidemiológicos apontou que houve a notificação de 15.181 casos de tuberculose no município de Recife, na tabela 1 é possível observar a distribuição dos casos no período de tempo estudado. A Tabela 1 mostra que o município apresentou crescimento contínuo no período de 2015 a 2017, sendo o ano de 2017 (2.666), o que apresentou o maior número de casos, após esse período, nota-se a ocorrência na diminuição dos casos em comparação com os anos anteriores, com o ano de 2020 (2.365) apresentando o menor número de casos. Contudo, essa informação pode não estar de acordo com a realidade da 
doença, já que segundo a OPAS (2021), devido a pandemia causada pelo novo coronavírus, no ano de 2020 houve uma redução no diagnóstico da doença, em comparação com o ano anterior, o que pode pôr em risco ações de combate contra a doença.

Tabela 1. Distribuição dos casos de tuberculose notificados no município do Recife no período de 2015 a 2020.

\begin{tabular}{ccc}
\hline ANO & CASOS & $\%$ \\
\hline 2015 & 2.534 & 16,7 \\
2016 & 2.568 & 16,9 \\
2017 & 2.666 & 17,6 \\
2018 & 2.481 & 16,3 \\
2019 & 2.567 & 16,9 \\
2020 & 2.365 & 15,6 \\
\hline Total & $\mathbf{1 5 . 1 8 1}$ & $\mathbf{1 0 0}$ \\
\hline
\end{tabular}

Fonte: Autores (2021), a partir de dados coletados do SINANWEB/DATASUS.

Na Tabela 2 é possivel observar a distribuição dos casos entre o sexo, sendo que os homens apresentaram 10.343 $(68,1 \%)$ e as mulheres $4.836(31,9 \%)$, também foram encontrados 2 casos que estavam como ignorados no banco de dados.

Tabela 2. Distribuição dos casos de tuberculose notificados no município do Recife no periodo de 2015 a 2020, distribuido por sexo

\begin{tabular}{ccc}
\hline SEXO & CASOS & \% \\
\hline Masculino & 10.343 & 68,1 \\
Feminino & 4.836 & 31,9 \\
Ignorado & 2 & 0,0 \\
\hline Total & $\mathbf{1 5 . 1 8 1}$ & $\mathbf{1 0 0}$ \\
\hline
\end{tabular}

Fonte: Autores (2021), a partir de dados coletados do SINANWEB/DATASUS.

Os dados apresentados no gráfico 2 corroboram com o estudo de Lima et. al. (2020) que em um estudo realizado na cidade de Imperatriz, no estado do Maranhão, que entre os anos de 2014 a 2015 foram registrados 129 casos de tuberculose no município, sendo que a maioria deles, $65,12 \%$, o mesmo que 84 casos foram registrados em homens, e as mulheres somaram 34,88\% (45). Outro estudo realizado por Oliveira et. al. (2018) no estado do Maranhão estudando os casos no período de 2012 a 2016 observou que 64,7\% (6.149) dos casos foram registrados em individuos do sexo masculino, enquanto apenas 35,3\% (3.343) foram nas mulheres. Segundo Barreto et al. (2020) o fato dos homens estarem mais propensos à exposição dos fatores de risco em comparação com as mulheres, associado com a desatenção pela própria saúde são possíveis justificativa para maior ocorrência da doença em homens. 
Tabela 3. Distribuição dos casos de tuberculose notificados no município do Recife no período de 2015 a 2020 , distribuído por faixa etária.

\begin{tabular}{ccc}
\hline IDADE & CASOS & $\%$ \\
\hline Branco/Ignorado & 3 & 0,02 \\
$<1$ & 98 & 0,65 \\
$1-4$ & 132 & 0,87 \\
$5-9$ & 126 & 0,83 \\
$10-14$ & 198 & 1,30 \\
$15-19$ & 719 & 4,74 \\
$20-39$ & 6.505 & 42,85 \\
$40-59$ & 5.011 & 33,01 \\
$60-64$ & 808 & 5,32 \\
$65-69$ & 603 & 3,97 \\
$70-79$ & 667 & 4,39 \\
$80<$ & 311 & 2,05 \\
\hline TOTAL & $\mathbf{1 5 . 1 8 1}$ & $\mathbf{1 0 0}$ \\
\hline
\end{tabular}

Fonte: Autores (2021), a partir de dados coletados do SINANWEB/DATASUS.

A Tabela 3 mostra que indivíduos que a maioria dos casos foram em pessoas na faixa etária de 20-39 anos, seguida da 30-59. Os dados apresentados vão de encontro com os estudos de Souza et. al. (2019) que em seus estudos sobre os casos de tuberculose na cidade de Sobral no estado do Ceará encontrou a maioria dos casos em indivíduos de 20 a 39 anos. Os estudos de Reis et al. (2013) também traz dados semelhantes aos observados na tabela 3. O fato de os adultos jovens serem mais acometidos pela doença indica que há uma transmissão recente da doença, essa característica é encontrada em países menos desenvolvidos (Longhi, 2013). Um fato importante a se observar é que o público idoso, aqueles com idade acima dos 60 anos, somaram juntos 2.389 casos, para Mesquita et. al. (2021) a associação entre o aumento da população idosa causada pela maior expectativa de vida com a alta quantidade de pessoas com tuberculose latente são fatores para a ocorrência da doença em idoso.

Tabela 4. Distribuição dos casos de tuberculose notificados no município do Recife no período de 2015 a 2020 , distribuído por forma clínica.

\begin{tabular}{ccc}
\hline FORMA & CASOS & $\%$ \\
\hline Branco/Ignorado & 3 & 0,0 \\
Pulmonar & 12.058 & 79,4 \\
Extrapulmonar & 2.377 & 15,7 \\
Pulmonar/Extrapulmonar & 743 & 4,9 \\
\hline TOTAL & $\mathbf{1 5 . 1 8 1}$ & $\mathbf{1 0 0}$ \\
\hline
\end{tabular}


Na Tabela 4 mostra que a maioria dos casos é da forma clínica pulmonar, esse achado vai de encontro com o estudo de Tavares et. al. (2020), que após a análise dos casos de TB no estado de Alagoas constatou que 86,32\% dos casos foram da forma pulmonar. Além de ser o primeiro em número de casos, a forma pulmonar é a que tem mais valor em estudo epidemiológico, já que, segundo Silva et. al. (2016) a transmissão ocorre quando um doente com a forma a TB pulmonar bacilífera expele no ar o Mycobacterium tuberculosis quando fala, espirra ou tosse. Um fato que pode explicar a maior ocorrência da forma pulmonar da TB é que a vacina usada para sua profilaxia, a BCG, não protege totalmente o aparecimento da forma pulmonar, mas garante uma proteção eficiente das formas graves da doença, que são as extrapulmonares.

A Tabela 5 mostra a associação da tuberculose com outras patologias e nos grupos vulneráveis, observa-se que o alcoolismo, tabagismo e AIDS são as três condições mais encontradas, contudo os dados apresentam um alto número de casos que estão ignorado/Branco, o que pode não conduzir com a atual realidade dos casos.

Tabela 5. Distribuição dos casos de tuberculose notificados no município do Recife no período de 2015 a 2020, distribuído por associação com outras patologias e grupos vulneráveis.

\begin{tabular}{cccc}
\hline VARIÁVEIS & CASOS & $\%$ \\
\hline AIDS & & & \\
Ignorado/Branco & 3.534 & 23,3 & 14,8 \\
Sim & 2.247 & 61,9 & \\
Não & 9.400 & \\
\hline
\end{tabular}

\begin{tabular}{ccc}
\hline ALCOOLISMO & & \\
Ignorado/Branco & 2.278 & 15,0 \\
Sim & 3.203 & 21,1 \\
Não & 9.700 & 63,9 \\
\hline
\end{tabular}

\section{DIABETES}

Ignorado/Branco

\section{TABAGISMO}

Ignorado/Branco

Sim

Não

PPL $^{1}$

Ignorado/Branco

Sim

Não

PSR $^{2}$
2.910

2.782

9.489
24,0

1.355

10.184
19,2

18,3

62,5 

Não
10.943
72,1

TOTAL

15.181

100

1 População Privada de Liberdade. ${ }^{2}$ População em Situação de Rua. Fonte: Autores (2021), a partir de dados coletados do SINANWEB/DATASUS.

O estudo da associação da Sida/TB é de suma importância, já que, segundo Prado et. al. (2011) desde a década de 1980 ela tem sido um dos principais fatores para o aparecimento da TB em países desenvolvidos e nos subdesenvolvidos também, ainda segundo o autor, o vírus causador da Sida alterou de forma significante a epidemiologia, história natural e a evolução clínica da TB, uma vez que pacientes que convivem com a associação Sida/TB apresenta um maior risco de apresentar a TB latente, além do aumenta da taxa de mortalidade em comparação de paciente apenas com a Sida. Além do mais, pacientes com HIV/Sida têm um risco 28 vezes maior de contrair a TB (Guia de vigilância de saúde, 2019).

O consumo crônico do álcool está associado com diversas complicações na vida do alcoólatra, entre eles, esse consumo de álcool causa uma alteração considerável na resposta imune do alcoólatra, deixando-o suscetível a sofrer de doenças que afetam o trato respiratório, entre dessas, a tuberculose. A importância do estudo da associação do alcoolismo e tuberculose está relacionado com o abandono ao tratamento, Oliveira et. al. (2012) em sua pesquisa sobre o abandono ao tratamento em uma unidade de saúde da família do Recife, mesma área pesquisada neste trabalho, constatou que dos 13 pacientes que abandonaram o tratamento, o alcoolismo esteve presente em 8 deles, ainda segundos os achados das autoras, a principal dificuldade está na questão dos pacientes terem que parar o consumo de álcool para o sucesso do tratamento. Essas questões tornam evidente que pacientes com essa condição necessitam de mais atenção por parte dos profissionais de saúde.

Segundo Seiscento (2012) pacientes diabéticos possui de 2,44 a 8,33 mais chance de contrair a TB daqueles que não são diabéticos, sendo aqueles que são insulinodependentes apresenta mais chance de riscos, também existe o aumento do risco de morte dos pacientes com a associação TB/diabetes daqueles que não apresentam a diabetes, ainda segundo o autor pacientes diabéticos frequentemente apresenta resistência ao tratamento. $\mathrm{O}$ fato de pacientes com diabetes mellitus serem mais suscetíveis a TB pode ser explicado pela significativa alteração que a doença causa no sistema imunológico, deixando o paciente suscetível a vários tipos de infecção, incluindo a TB (Sousa et. al. 2021).

O tabagismo é um dos principais fatores de riscos para a ocorrência de cânceres, doenças circulatórias e respiratórias, se configurando como um dos principais causador de morte por causas evitáveis no mundo, é esperado que até 2050 haja um crescimento dos casos de tuberculose causada pelo tabagismo (Oliveira et. al. 2020). Para Silva et. al. (2018) a fumaça do cigarro causa uma disfunção ciliar, a uma resposta imune reduzida e principalmentes a defeitos na resposta imune gerada por macrófagos, relacionada principalmente a baixa síntese de citocinas com a IL-12 e a TNF- $\alpha$, o autor ainda comenta que a um aumento significativo da taxa de mortalidade entre os fumantes em comparação com os não fumantes.

O estudo sobre como a tuberculose atinge as populações vulneráveis é de suma importância, principalmente as populações que são privadas de liberdade ou vivem em situação de rua, uma vez que, segundo Aguiar et. al. (2021) essas populações têm respectivamente 28 e 56\% mais chances de contrair a TB. Segundo Borges et. al. (2021) a TB é uma doença persistente no sistema carcerário brasileiro, já que o diagnóstico tardio, rotatividade de presos, superlotação e as condições precárias dos presídios, entre outros fatores, contribuem para sua ocorrência. Em relação à população em situação de rua existe um imenso desafio de ações voltadas para o controle da TB, causada pela complexidade da garantia de uma assistência de saúde eficiente para essa população (Hino et. al. 2021).

A Tabela 6 mostra o modo de entrada dos casos e a situação do encerramento dos casos de TB. 
Tabela 6. Distribuição dos casos de tuberculose notificados no município do Recife no período de 2015 a 2020 , distribuído por modo de entrada e encerramento.

\begin{tabular}{ccc}
\hline VARIÁVEIS & CASOS & $\%$ \\
\hline MODO DE ENTRADA & 10.912 & 71,9 \\
Caso novo & 1.196 & 7,9 \\
Recidiva & 1.650 & 10,9 \\
Reingresso após abandono & 110 & 0,6 \\
Não sabe & 559 & 3,7 \\
Transferência & 754 & 5,0 \\
Pós-óbito & & 6,2 \\
\hline SITUAÇÃO DE ENCERRAMENTO & 943 & 47,6 \\
Ignorado/Branco & 7.219 & 12,2 \\
Cura & 1.852 & 8,9 \\
Abandono & 1.354 & 8,3 \\
Óbito por tuberculose & 1.261 & 15,0 \\
Óbito por outras causas & 2.282 & 0,7 \\
Transferência & 111 & 0,7 \\
TB-DR & 101 & 0,1 \\
Mudança de esquema & 13 & $\mathbf{1 0 0}$ \\
Falência & 45 & \\
Abandono primário & $\mathbf{1 5 . 1 8 1}$ & \\
\hline TOTAL & & \\
\hline & & \\
\hline & & \\
\hline
\end{tabular}

Fonte: Autores (2021), a partir de dados coletados do SINANWEB/DATASUS.

Os achados apresentados na tabela 6 são semelhantes a outros achados na literatura, como o de Silva et. al. (2015), que analisando os casos de tuberculose no estado de Alagoas, observou que 83,2\% do modo de entrada foram de novos casos, semelhantes também com os de Coutinho et. al. (2012), que observou que a maioria das entradas por tuberculose na cidade de João Pessoa era de casos novos (79,9\%). Possíveis fatores que explicam esse fato seriam a baixa qualidade dos serviços de saúde, falhas no detectamento da doença e também a deficiência no acompanhamento de pessoas que estão em tratamento, que leva à desistência do tratamento por parte dos doentes (Santos et. al. 2020).

Os dados apresentados na tabela 6 ainda mostram que o maior número de encerramentos dos casos foi por cura, com $7.219(47,6 \%)$ dos casos, outro dado em evidência é o de abandono, que esteve presente em 1.852 (12,2\%). Esses valores estão abaixo do recomendado pela WHO, que indica para que haja sucesso ao combate da TB que haja um índice de cura igual ou superior a $85 \%$, e que os de abandono somem menos que 5\% (BRASIL 2017). Esse elevado casos de abandono é preocupante, pois segundo Mendes. et. al. (2021) o abandono ao tratamento causa um maior carga bacilar pelo paciente, favorecendo assim uma maior transmissibilidade da doença, maior morbimortalidade e também possibilita o aparecimento de bacilos resistentes. 


\section{Conclusão}

A partir da análise dos dados, destaca-se que na cidade do Recife, no período de 2015 a 2020, a TB ainda se configura como uma doença endêmica. Salienta-se que ela atinge mais os homens, de forma significativa, com a faixa etária de 20 a 39 anos, apresentando prevalência da forma pulmonar. A maioria dos casos se apresenta como quadro novo, o que demonstra que a TB ainda está bastante prevalente no município. O índice elevado de abandono associado com o baixo índice de cura, também são fatores preocupantes.

Os resultados encontrados demonstram a necessidade de ações mais firmes voltadas para o controle da TB, como a educação continuada voltada para os profissionais de saúde, o acompanhamento de perto de pessoas que estão em tratamento, buscando ferramenta de educação em saúde que estimule o paciente a prosseguir com tratamento, além de um forte programa de rastreio de casos da doença.

Pelo fato de Recife está frequentemente presente na lista dos municípios prioritários a ocorrência da TB em Pernambuco, espera-se que esse trabalho influencie na realização de novos estudos sobre a doença na cidade. Seria de extrema importância que esses estudos abrangessem, principalmente na compreensão dos fatores que levam o paciente a abandonar o tratamento, para que os gestores de saúde possam elaborar políticas públicas que possam diminuir o abandono ao tratamento da TB no município.

\section{Referências}

Aguiar, F. H. S., Calhau, G. S., Lachtim, S. A. F., Pinheiro, P. N. C., Arcêncio, R. A., \& Freitas, G. L., (2021). Perfil da tuberculose em populações vulneráveis: pessoas privadas de liberdade e em situação de rua. Revista De Ciências Médicas E Biológicas, 20(2), 253-258. https://doi.org/10.9771/cmbio.v20i2.43513

Barreto, M. T. S., Santos, G. M. dos, Monteiro, M. J. de S. D., Jesus, R. L. R. de, Barbosa, G. S., \& Oliveira, V. A. de. (2020). Epidemiologia da tuberculose em um estado do nordeste brasileiro. Research, Society and Development, 9(7), e52973643. https://doi.org/10.33448/rsd-v9i7.3643

Bertolozzi, M. R., Takahashi, R. F., Hino, P., Litvoc, M., \& França, F. O. S., (2014). O controle da tuberculose: um desafio para saúde pública. Revista de medicina, 93(2), 83-89. https://doi.org/10.11606/issn.1679-9836.v93i2p83-89

Borges, T. M., \&Witt, N. G. P. M., (2021). A tuberculose e a população privada de liberdade: os casos notificados em joaçaba/sc, no período de 2017 a 2019. Caderno Intersaberes, 10(26), 4-18, 2021. https://www.cadernosuninter.com/index.php/intersaberes/article/view/1851

Brasil. Ministério da Saúde. Secretaria de Vigilância em Saúde. (2017). Indicadores prioritários para o monitoramento do Plano Nacional pelo Fim da Tuberculose como Problema de Saúde Pública no Brasil. Boletim Epidemiológico. 48(8), 1-11.

Brasil. Ministério da Saúde (2020). O que é a tuberculose? Governo Federal. https://www.gov.br/saude/pt-br/assuntos/saude-de-a-a-z/t/tuberculose

Brasil. Ministério da Saúde (2019). Guia de vigilância em saúde, volume, único (3a ed). https://bvsms.saude.gov.br/bvs/publicacoes/guia_vigilancia_saude_3ed.pdf

Campos, R. \& Pianta, C. (2001). Tuberculose: histórico, epidemiologia e imunologia, de 1990 a 1999, e co-infecção TB/HIV, de 1998 a 1999, Rio Grande do Sul - Brasil. Bol. da Saúde, v. 15, n. 1, 2001. http://www.boletimdasaude.rs.gov.br/conteudo/1151/tuberculose:-historico,-epidemiologia-e-imunologia,-de1990-a-1999,-e-co-infeccao-tb-hiv,-de-1998-a-1999,-rio-grande-do-sul-?-brasil

Coutinho, L. A. S. de A., Oliveira, D. de S., Souza, G. de F., Fernandes Filho, G. M. C., \& Saraiva, M. G. (2012). Perfil epidemiológico da tuberculose no município de João Pessoa - PB, entre 2007 - 2010. Revista Brasileira De Ciências Da Saúde, 16(1), 35-42. https://periodicos.ufpb.br/ojs/index.php/rbcs/article/view/10172

Espirito Santo. Secretaria de Estado da Saúde do Espirito Santo. Tuberculose. Governo do Estado do Espirito Santo. https://saude.es.gov.br/neve-tuberculose

Hijjar, M. A., Gerhardt, G., Teixeira, G. M., \& Procópio, M. J., (2007). Retrospecto do controle da tuberculose no Brasil. Rev Saúde Pública 2007;41(Supl. 1):50-58. https://doi.org/10.1590/S0034-89102007000800008

Hino, P., Yamamoto, T. T., Bastos, S. H., Beraldo, A. A., Figueiredo, T. M. R. M., \& Bertolozzi, M. R., (2021). Tuberculose na população de rua: revisão sistemática. Rev Esc Enferm USP, 2021;55:e03688. https://doi.org/10.1590/S1980-220X2019039603688

Hochman, B., Nahas, F. X., Filho, R. S. O., \& Ferreira, L. M., (2005). Desenhos de pesquisas. Acta cirúrgica brasileira - 20 (2). https://www.scielo.br/j/acb/a/bHwp75Q7GYmj5CRdqsXtqbj/?format=pdf\&lang=pt

Instituto Brasileiro de Geografia e Estatística (2021). Cidades e Estados, Recife. https://www.ibge.gov.br/cidades-e-estados/pe/recife.html

Maciel, S. M., Mendes, P. D., Gomes, A. P., \& Batista, R. S. (2012). A história da tuberculose no Brasil: os muitos tons (de cinza) da miséria. Rev Bras Clin Med. São Paulo, 2012 mai-jun;10(3):226-30. http://files.bvs.br/upload/S/1679-1010/2012/v10n3/a2886.pdf 
Lima, P. H. S., Santos, F. S., Santos, L. H., Nunes, S. E. A., Santos, L. F. S., Pascoal, L. M., Sousa, G. G. S., Firmo, W. C. A., \& Neto, M. S. (2020). Perfil epidemiológico dos casos de tuberculose em Imperatriz, Maranhão, Brasil. Research, society and development, 9(7), e170973998. http://dx.doi.org/10.33448/rsdv9i7.3998

Longhi, R. M. P. (2013). Fatores de risco associados ao desenvolvimento de tuberculose na população urbana no município de Dourados, MS. 63 f. Dissertação (Mestrado Profissional em Saúde Pública) - Escola Nacional de Saúde Pública Sergio Arouca, Fundação Oswaldo Cruz, 2013. https://www.arca.fiocruz.br/handle/icict/24544

Mesquita, C. R., Lima, K. V. B., Guimarães, R. J. P. S., Santos, B. O., Rodrigues, L. H. A., Costa, R. J, F., Santos, B. S. O., \& Lúdice, T. N. S., (2021). Analise retrospectiva de casos de tuberculose em idosos. Revista Brasileira Em Promoção da Saúde, 34. https://doi.org/10.5020/18061230.2021.11117

Mendes, R. C., da Silva, W. C., da Silva, C. O., Soares, A. N., Melo, K. C., dos Santos Anjos, D., \& Conceição, L. L. (2021). Aspectos epidemiológicos da tuberculose no Município de Caxias, do Estado do Maranhão. Research, Society and Development,10(3), e39810313284. https://doi.org/10.33448/rsdv10i3.13284

Oliveira, J. F., \& Antunes, M. B. C., (2012). Abandono anunciado ao tratamento da tuberculose em uma unidade de saúde da família do Recife - A perspectiva do usuário. Rev APS, 15(1): 4-13. https://periodicos.ufjf.br/index.php/aps/article/view/14930

Oliveira, M. S. R., Sousa, L. C., Baldoino, L. S., Alvarenga, A. A., Silva, M. N. P., Elias, S. C. G., Macedo, L. S., \& Silva, M. R. S. (2018). Perfil epidemiológico dos casos de tuberculose no estado do Maranhão nos anos de 2012 a 2016. Rev Pre Infec e Saúde. 4, 6896. https://doi.org/10.26694/repis.v4i0.6896

Oliveira, R. L., Azevedo, L. S., Macêdo, E. S., Aguiar, M. L. P., Abreu, A. S., Privado, L. B., Nascimento, L. H. B., Azevedo, H. Z. V. P., \& Azevedo, A. P., (2020). Relatos de uso de tabaco, álcool e drogas ilícitas entre pacientes em tratamento para tuberculose. Braz. J. Hea. Ver., 3(5), 14866-14877. https://doi.org/10.34119/bjhrv3n5-278

OPAS (2021). Diagnóstico de novos casos de tuberculose caiu entre 15\% e 20\% nas Américas em 2020 devido à pandemia. https://www.paho.org/pt/noticias/243-2021-diagnostico-novos-casos-tuberculose-caiu-entre-15-e-20-nas-americas-em-2020

Prado, T. N., Caus, A. L., Marques, M., Maciel, E. L., Golub, J. E., \& Miranda, A. E., (2011). Perfil epidemiológico de pacientes adultos com tuberculose e AIDS no estado de Espirito Santo, Brasil: relacionamento dos bancos de dados de tuberculose e AIDS. Jornal Brasileiro de Pneumologia, 37, 93-99. https://www.scielo.br/j/jbpneu/a/fZvBBgjGCfsxWHshSL5fgwR/?format=pdf\&lang=pt

$\begin{array}{lllllll}\text { Pernambuco (2019). Secretaria } & \text { Estadual } & \text { de } & \text { Saúde. } & \text { Programa } & \text { Sanar, }\end{array}$ http://portal.saude.pe.gov.br/sites/portal.saude.pe.gov.br/files/plano_sanar_2_edicao_29.08.17.pdf

Reis, D. C., Almeida, T. A. C., Quites, H. F. O., \& Sampaio, M. M., (2013). Perfil epidemiológico da tuberculose no Município de Belo Horizonte (MG), no período de 2002 a 2008. Revista brasileira de epidemiologia, 2013, v. 16, n. 03, pp. 592-602. https://doi.org/10.1590/S1415-790X2013000300004

Seiscento, M., (2012). Tuberculose em situações especiais: HIV, diabetes mellitus e insuficiência renal. Pulmão RJ, (21), 23-6. http://www.sopterj.com.br/wpcontent/themes/_sopterj_redesign_2017/_revista/2012/n_01/06.pdf

Silva, D. S., Torrico, M. M., Duarte, R., Galvão, T., Bonini, E. H., Arbex, F. F., Arbex, M. A., Augusto, V. M., Rabahi, M. F., \& Mello, F. C. Q., (2018). Fatores de risco para tuberculose: diabetes, tabagismo, álcool e uso de outras drogas. J Bras Pneumol; 44(2): 145-152. http://dx.doi.org/10.1590/S180637562017000000443

Silva, E. G., Vieira, J. D. S., Cavalcante, A. L., Santos, L. G. M. L., Rodrigues, A. P. R. A., \& Cavalcante, T. C. S., (2015). Perfil epidemiológico da tuberculose no estados de Alagoas-AL de 2007 a 2012. Caderno de Graduação - Ciências Biológicas e da Saúde - UNIT - ALAGOAS, 3(1), 31-46. https://periodicos.set.edu.br/fitsbiosaude/article/view/2352

Silva, É. K., \& Silva, G. A., (2016). O sentido de vivência a tuberculose: um estudo sobre representações sociais das pessoas em tratamento. Revista de saúde coletiva 26(4), 1233-1247. https://doi.org/10.1590/S0103-73312016000400009

Sousa, G. G. S., Pascoal, L. M., Costa, A. C. P. J., Santos, F. S., Santos, L. H., Arcêncio, R. A., \& Neto, M. S., (2021). Tendência e fatores associados à comorbidade tuberculose-diabetes mellitus em um município do nordeste brasileiro. Rev Bras Enferm, 74(3):e20201238. https://doi.org/10.1590/0034-71672020-1238

Souza, A. C., Custódio, F. R., \& Melo, O. F., (2019). Cenário epidemiológico da tuberculose no município de Sobral (CE) entre os anos de 2013 e 2017. Revista eletrônica acervo saúde, (23), e445. https://doi.org/10.25248/reas.e445.2019

Tavares, C. M., Cunha A. M. S., Gomes, N. M. C., Lima, A. B. A., Santos, I. M. R., Acácio, M. S., Santos, D. M., \& Souza, C. D. F., (2020). Tendência e caracterização epidemiológica da tuberculose em Alagoas, 2007-2016. Caderno Saúde Coletiva, 28(1), 107-115. https://doi.org/10.1590/1414$462 X 202028010381$

World Health Organization (WHO, 2020). Global Tuberculosis Report 2020. https://www.who.int/publications/i/item/9789240013131/ 